\title{
INOVAÇÃO DE PROCESSOS - UM ESTUDO COMPARATIVO SOBRE SUA IMPLEMENTAÇÃO
}

\section{PROCESSES INNOVATION - A COMPARATIVE STUDY ABOUT ITS IMPLEMENTATION}

\author{
Débora Soares $^{1}$; Rogerio Valle ${ }^{2}$; Roquemar Baldam ${ }^{3}$; Telma Ragonezi ${ }^{4}$ \\ ${ }^{1}$ COPPE/UFRJ - Rio de Janeiro - Brasil dmacedo@pep.ufrj.br \\ ${ }^{2}$ COPPE/UFRJ - Rio de Janeiro - Brasil valle@pep.ufrj.br \\ ${ }^{3}$ COPPE/UFRJ - Rio de Janeiro - Brasil roquemar@pep.ufrj.br \\ ${ }^{4}$ ADM Brasil Consulting - Rio de Janeiro - Brasil telmaragonezi@admbrasilconsulting.com.br
}

\begin{abstract}
Resumo
Num ambiente globalizado de intensa competição e de mudanças constantes e complexas, caracterizadas por exigências cada vez maiores em termos de rapidez, qualidade, baixo custo, flexibilidade e satisfação do cliente, o sucesso de uma organização reside na sua capacidade de responder adequadamente a essas exigências, através de inovações contínuas em seus produtos, processos e serviços. Neste contexto, este trabalho analisa as premissas, as motivações e os impactos causados pela Inovação de Processos, nas organizações que utilizaram esta estratégia como forma de obter um diferencial competitivo no mercado. Para alcançar os objetivos pretendidos, apresenta-se uma revisão bibliográfica sobre o tema e em seguida comparam-se os resultados depreendidos de alguns estudos de casos.
\end{abstract}

Palavras-chave: mudanças organizacionais; inovação de processos; organizações.

\section{Introdução}

A partir da década de 80, as organizações vêm sofrendo pressões crescentes, decorrentes de mudanças radicais e contínuas no ambiente organizacional. Segundo Nadler \& Tushman (2000), três pontos críticos do ambiente organizacional influenciam o funcionamento das organizações. Primeiro, o ambiente faz exigências à organização; ele pode, por exemplo, exigir certos produtos ou serviços, com certos níveis de qualidade e quantidade. Segundo, o ambiente pode impor restrições à ação organizacional, pode limitar as atividades a que uma organização se dedica. Essas restrições, segundo os autores, vão desde as impostas pela escassez de capital ou tecnologia até a proibição pelos regulamentos governamentais. Terceiro, o ambiente oferece oportunidades a serem exploradas pela organização. 
Diante desse cenário, o sucesso de uma organização depende fundamentalmente de sua capacidade de resposta a essas pressões do ambiente. Conforme destaca Senge (2000), o sucesso da organização dependerá do grau em que ela conseguirá sustentar seus processos de mudança e transformação. De acordo com Daft (2002), tais mudanças podem se focalizar em: mudanças nos produtos e serviços; mudanças nos processos e na tecnologia; mudanças na estratégia e na estrutura organizacional; e mudanças na cultura organizacional.

Nesse novo contexto de mudança nas organizações, diversos autores citam que a inovação de processos - termo atualmente mais empregado à antiga reengenharia de processos de negócios, assume papel de destaque para o seu sucesso. Hammer (1997) destaca que, a partir de 1985, muitas empresas perceberam que seus métodos tradicionais de desenvolvimento de produto, fabricação, venda e assistência técnica já não se mostravam compatíveis com a concorrência global e com clientes cada vez mais exigentes, assim como as ferramentas e técnicas disponíveis para melhorar a qualidade e a satisfação dos clientes e reduzir custos não estavam apresentando resultados satisfatórios. Muitas dessas empresas decidiram implementar a inovação de processos. Davenport (1994) observa que a inovação de processos é um veículo fundamental para a implementação de estratégias de redução de custo, aumento da velocidade e satisfação do cliente, tornando-se importante fonte de vantagem competitiva para as organizações.

Por outro lado, a aplicação da inovação de processos apresentou uma série de problemas nas organizações. Porter (1997) salienta que muitas organizações aplicaram a inovação de processos indiscriminadamente, isto é, movidas mais pelo entusiasmo do que pela compreensão de como aplicar seu conceito, o que levou a uma alta taxa de fracassos. Manganelli (1995) ressalta que uma das razões pelas quais os projetos de inovação de processos fracassam é a sua interpretação equivocada como automação, reorganização ou downsizing.

Considerando os aspectos supramencionados, infere-se que a inovação de processos é um tema bastante controvertido, motivando que se analisem as questões mais relevantes sobre sua implementação nas organizações.

Com esse intuito, este estudo tem como objetivo verificar em relação à inovação de processos: as principais motivações (ou objetivos pretendidos) para a adoção desta técnica; com quais aspectos ela contribui para o alcance destes objetivos; os principais fatores que facilitam sua implementação (com destaque para a metodologia utilizada e o gerenciamento da mudança); e as influências desta técnica no desempenho das organizações. 


\section{A inovação de processos}

\subsection{Conceitos básicos}

Davenport (1994) conceitua a inovação como uma alteração de processo que provoque uma mudança importante e radical, ou seja, esta técnica modifica a estrutura das atividades do processo, visando "resultados visíveis e drásticos". Sob esta ótica, o autor salienta que a reengenharia de processos distingue-se da melhoria de processos, que visa um nível mais superficial de mudanças.

Segundo Hammer e Champy (1994), a reengenharia de processos de negócios significa uma reestruturação radical dos processos, com o objetivo de alcançar melhorias drásticas em indicadores de desempenho como custos, qualidade, atendimento ao cliente e velocidade.

Dos conceitos citados pelos autores, conclui-se que a reengenharia de processos de negócios é um esforço organizado para reestruturar integralmente os processos inerentes aos tipos de negócios de uma organização, visando proporcionar ganhos significativos em indicadores de desempenho, tais como: custos, qualidade, velocidade e satisfação do cliente, bem como obter vantagem competitiva.

\subsection{As principais motivações para a adoção da inovação}

Davenport (1994) salienta que a inovação de processos sustenta estratégias de: satisfação do cliente - redução do tempo de ciclo do processamento do pedido, melhoria da qualidade do produto/serviço e agilidade da solução de reclamações; redução de custos e do preço do produto/serviço - eliminação de atividades que oneram os custos dos produtos e serviços e repasse dos ganhos ao cliente; inovação de produtos - redução do tempo de ciclo de desenvolvimento e maior retorno sobre o investimento em inovação; e aumento da receita, melhoria da lucratividade e aumento do percentual de participação do mercado - como conseqüência das estratégias anteriores.

Segundo Morris e Brandon (1994), a inovação de processos é adotada quando uma organização busca alcançar metas importantes para o seu sucesso, tais como: redirecionar a operação - significa eliminar operações redundantes, melhorar o fluxo do trabalho e das informações e aprimorar os sistemas de apoio, proporcionando maior eficiência, flexibilidade e qualidade às operações; reduzir os custos - através da medição e da avaliação da eficácia dos esforços da inovação, podendo contemplar os custos de mão-de-obra (eliminação de atividades desnecessárias), informação (relacionados a obtenção, guarda, processamento e disseminação das informações para a tomada de decisões) e materiais (melhor integração entre a empresa e seus fornecedores, resultando em menores custos de aquisição); melhorar a qualidade - agrega valor 
aos produtos/serviços e ao cliente, reduzindo desperdícios e custos e aumentando a confiabilidade de atendimento do pedido do cliente e dos projetos de desenvolvimento de novos produtos/serviços; aumentar a receita - mediante a alteração de fatores como a elevação da quantidade produzida decorrente da diminuição dos custos e conseqüente repasse ao preço do produto/serviço, redução do tempo de ciclo de fabricação e aumento da velocidade de inovação nos produtos/serviços; melhorar a orientação voltada para o cliente - a percepção que o cliente tem da empresa e dos seus produtos está intimamente relacionada com o diferencial dos serviços que ela oferece; e melhorar a lucratividade - a redução de custos, o aumento da receita e a melhoria da satisfação do cliente levam ao incremento da lucratividade.

Analisando o descrito acima, pode-se dizer que as motivações (ou objetivos pretendidos) que levam as organizações a adotarem a inovação de processos estão relacionadas com a implementação de estratégias que visam melhorar seu desempenho e conquistar vantagem competitiva.

\subsection{Os principais fatores que facilitam a implementação da inovação}

Habilitadores (ou facilitadores) são condições que, quando satisfeitas, facilitam a implementação de uma determinada mudança, permitindo que ela atinja seus objetivos.

Chiavenato (1998) cita que a inovação necessita de habilitadores para alcançar os objetivos propostos, assim como Davenport (1994) cita que os projetos de reengenharia de processos de negócios necessitam de "habilitadores específicos" para alcançar os resultados desejados.

Bergamaschi (1999) aborda o conceito de fatores críticos de sucesso em projetos de reengenharia de processos de negócios, destacando que certos fatores considerados críticos, quando presentes na implementação da inovação, facilitam de forma relevante a implementação desta técnica.

Morris e Brandon (1994) identificam como habilitadores da inovação de processos: comprometimento da alta administração - fornecer todo o apoio necessário e assumir responsabilidades para que a inovação seja bem sucedida; alinhamento com a estratégia - a inovação deve ser utilizada como apoio para a implementação das estratégias que possam agregar valor para o cliente; metodologia para a inovação - sua implementação deve ser conduzida segundo uma abordagem sistemática; gerenciamento da mudança - refere-se a um esforço coordenado para minimizar resistências; suporte de Tecnologia da Informação - a Tecnologia da Informação pode melhorar a eficiência dos processos de negócios; e equipes de engenharia devem ser utilizadas equipes multidisciplinares e autogeridas para serem responsáveis pela condução dos projetos de inovação. 
É importante ressaltar que, no que se refere aos habilitadores da inovação de processos, a metodologia utilizada e o gerenciamento da mudança são fatores críticos de sucesso. Tais habilitadores são analisados a seguir:

\subsubsection{Metodologia utilizada na implantação da inovação}

Davenport (1994) cita a falta de uma metodologia adequada como uma das razões para o fracasso das iniciativas da inovação de processos em diversas organizações.

Morris e Brandon (1994) ensinam que uma abordagem sistemática para a inovação deve abranger as seguintes fases: definir o posicionamento da organização - analisar o ambiente externo para detectar oportunidades e ameaças para o negócio, confrontar essa análise com a atual situação do ambiente interno da organização, identificar seus pontos fortes e fracos e definir objetivos, estratégias e prazos para que a empresa aumente sua competitividade no mercado; estabelecer um novo ambiente na empresa - identificar as mudanças a serem implementadas, tendo em vista o novo posicionamento estratégico da organização e substituir os atuais paradigmas que não se adequarem a esse novo posicionamento por outros que possibilitem obter uma vantagem competitiva sustentável; mapear os processos atuais - levantar informações sobre os processos existentes na empresa para compreender suas funcionalidades, níveis de desempenho e interligações internas e externas, identificar quais os processos vitais para o negócio e, partindo destas informações, definir a ordem de prioridade dos processos a serem aprimorados; redesenhar o processo - o mapeamento dos processos atuais (item anterior) fornece as bases para que o processo-alvo da inovação seja analisado em detalhes e um novo modelo de processo seja desenhado; e implantar e monitorar o novo processo - deve ser providenciada a infra-estrutura necessária para que o novo processo entre em operação, abrangendo aspectos como aquisição dos recursos de hardware e software, modificações em lay-outs e treinamento das pessoas envolvidas na estrutura organizacional.

\subsubsection{Gerenciamento da mudança}

Pereira (1999) destaca que as mudanças ocorrem de forma contínua e independente da nossa vontade, implicando a necessidade de incorporar coisas novas e renunciar a outras, de forma que as mudanças acabam gerando resistências que devem ser administradas.

Tendo em vista que a inovação de processos contempla mudanças altamente complexas e de grande impacto sobre a organização - especialmente sobre as pessoas - é necessário que ela seja conduzida de acordo com um esforço coordenado que promova a mudança de atitudes e minimize as resistências às mudanças, ou seja, um gerenciamento coordenado da mudança. 
De acordo com Lewin (1947), para que o processo de mudança nas organizações seja bem sucedido, deve seguir três etapas: descongelamento do padrão atual de comportamento - as antigas idéias e práticas são desfeitas e desaprendidas, dando lugar a outras novas, que deverão ser aprendidas, sendo fundamentais o entendimento e a aceitação da mudança pelas pessoas; mudança - novas idéias e práticas são experimentadas e aprendidas, de modo que as pessoas passam a pensar e agir de acordo com a nova maneira; e recongelamento - as novas idéias e práticas aprendidas são incorporadas definitivamente ao comportamento das pessoas.

Quando as forças positivas superam as negativas, a mudança é bem sucedida; caso contrário, a mudança não ocorre, retornando-se à situação antiga. Para que a mudança realmente aconteça, devem ser adotados mecanismos que aumentem as forças positivas e diminuam as negativas.

Conforme convenientemente define Covey (1996), tais mecanismos devem estar centrados nos seguintes aspectos: conscientização - convencer as pessoas sobre a necessidade e a direção da mudança; envolvimento - fazer com que as pessoas compartilhem a missão da organização e analisem e aceitem os impactos da mudança; segurança interior - criar um ambiente seguro, de forma que as pessoas considerem a mudança como uma oportunidade de crescimento e não como uma ameaça; responsabilidade pelos resultados - convencer as pessoas sobre a sua responsabilidade pelo desempenho da organização e pelos resultados da mudança; mente aberta incentivar as pessoas a serem receptivas às novas idéias e à inovação contínua; sinergia - estimular o trabalho em equipe, para que ocorra uma soma de conhecimentos e se obtenha a melhor alternativa de solução para os problemas; e interesse coletivo - estimular as pessoas a colocarem os interesses gerais da empresa acima dos individuais, visando à interação do grupo.

\subsection{As influências da inovação de processos no desempenho das organizações}

Baseando-se no estudo de várias empresas norte-americanas que adotaram a inovação, Hammer e Champy (1994) identificaram algumas características que são freqüentemente encontradas nestas organizações: vários trabalhos (empregos) são combinados em apenas um; os trabalhadores tomam as decisões; os passos no processo são realizados na ordem natural; os processos têm múltiplas versões; o trabalho é realizado onde ele faz mais sentido; as verificações e os controles são reduzidos; a reconciliação de informações é minimizada; um gerente de caso (case manager) provê um único ponto de contato com o cliente; e as operações centralizadas são descentralizadas.

Quanto às mudanças efetuadas pelas empresas que implementaram a inovação de processos, estes autores também observaram os seguintes pontos: as unidades de trabalho se alteram de departamentos funcionais para times/grupos de processo; o tipo de trabalho realizado pelas pessoas 
muda de tarefas simples para um trabalho multidimensional; o papel da força de trabalho evolui de um estado controlado para uma situação autônoma (empowerment); a preparação para o trabalho se altera de treinamento para educação; as medidas de desempenho e a forma de compensação das pessoas mudam do foco na atividade/tempo para o foco na obtenção de resultados; os critérios de encarreiramento também sofrem alterações, passando de promoções baseadas no desempenho para promoções baseadas nas habilidades; o sistema de valores da organização passa de protetivo para o produtivo; o papel gerencial, visto anteriormente como de supervisão, passa a ser de auxiliar, de mentor; as estruturas organizacionais deixam de ser altamente hierarquizadas e passam a ser mais planas; e os executivos assumem a função de líderes ao invés de controladores de índices de desempenho econômico.

Como mencionado anteriormente, a inovação de processos demanda que a empresa opere sob um novo conjunto de paradigmas organizacionais, humanos e tecnológicos.

\subsection{Considerações importantes sobre a inovação de processos}

Aqui, apresentam-se diversos conceitos relevantes e pertinentes à inovação de processos, extraídos de artigos indexados, como parte integrante da pesquisa bibliográfica realizada neste trabalho:

\subsubsection{As melhores práticas da inovação de processos}

Reijers \& Mansar (2005) definem as melhores práticas da inovação de processos com base na experiência que obtiveram em grandes organizações ou firmas de consultoria com implementações de inovação. Estas melhores práticas direcionam-se para: clientes, com foco na melhoria de contatos; operação de processos de negócio, com foco em como implementar o fluxo de trabalho; organização, considerando tanto sua estrutura quanto os recursos envolvidos (tipos e número); informação, descrevendo melhores práticas referentes à tecnologia que os processos de negócios utilizam; e ambiente externo, tentando melhorar a colaboração e a comunicação entre os elementos a cadeia de valor da organização.

\subsubsection{Elementos de mudança organizacional na inovação de processos}

Teng et al. (1996) identificou, segundo sua visão, os elementos da mudança organizacional que devem ser monitorados para viabilizar o sucesso de uma implementação de inovação, mediante o encadeamento de cinco aspectos apresentados a seguir e ilustrados na figura $\mathbf{1}$ a seguir: motivação organizacional para a inovação - promoção de um ambiente de inovação, 
planejamento da estratégia corporativa e planejamento da estratégia de TI; iniciando a mudança de processo - analisar a dinâmica da mudança, formular a estratégia de mudança, assegurar o comprometimento dos stakeholders e estruturar o processo de mudança; selecionar os habilitadores de mudança - habilitadores da estrutura organizacional, habilitadores do sistema gerencial, habilitadores de recursos humanos e habilitadores da Tecnologia da Informação; gerenciar a implementação da mudança - analisar os problemas de implementação, gerenciar a política de implementação, aplicar as técnicas de implementação e avaliar as condições de implementação; e direções da mudança organizacional - a dimensão estrutural, a dimensão gerencial e a dimensão dos recursos humanos.

Figura 1 - Inovação de Processos - Elementos de mudança organizacional

\begin{tabular}{|c|c|c|c|c|}
\hline $\begin{array}{c}\text { Motivação } \\
\text { organizacional } \\
\text { para a RPN }\end{array}$ & $\begin{array}{l}\text { Iniciando o } \\
\text { Processo de } \\
\text { Mudança }\end{array}$ & $\begin{array}{c}\text { Selecionando os } \\
\text { Habilitadores } \\
\text { da Mudança }\end{array}$ & $\begin{array}{c}\text { Gerenciando a } \\
\text { Implementação } \\
\text { da Mudança }\end{array}$ & $\begin{array}{c}\text { Direções da } \\
\text { Mudança } \\
\text { Organizacional } \\
\end{array}$ \\
\hline $\begin{array}{l}\text { - Promoção de } \\
\text { um ambiente de } \\
\text { inovação }\end{array}$ & $\begin{array}{l}\text { - Analisando a } \\
\text { dinâmica da } \\
\text { mudança }\end{array}$ & $\begin{array}{l}\text { - Habilitadores } \\
\text { da estrutura } \\
\text { organizacional }\end{array}$ & $\begin{array}{l}\text { - Analisando os } \\
\text { problemas de } \\
\text { implementação }\end{array}$ & $\begin{array}{l}\text { - A dimensão } \\
\text { estrutural }\end{array}$ \\
\hline $\begin{array}{l}\text { - Planejamento } \\
\text { da estratégia } \\
\text { corporativa }\end{array}$ & $\begin{array}{l}\text { - Formulando a } \\
\text { estratégia de } \\
\text { mudança }\end{array}$ & $\begin{array}{l}\text { - Habilitadores } \\
\text { do sistema de } \\
\text { gerenciamento }\end{array}$ & $\begin{array}{l}\text { - Gerenciando a } \\
\text { política de } \\
\text { implementação }\end{array}$ & $\begin{array}{l}\text { - A dimensão } \\
\text { gerencial }\end{array}$ \\
\hline $\begin{array}{l}\text { - Planejamento } \\
\text { da estratégia de } \\
\text { TI }\end{array}$ & $\begin{array}{l}\text { - Assegurando o } \\
\text { comprometiment } \\
\text { o dos } \\
\text { stakeholders } \\
\text { - Estruturando o } \\
\text { processo de } \\
\text { mudanca }\end{array}$ & $\begin{array}{l}\text { - Habilitadores } \\
\text { de recursos } \\
\text { humanos } \\
\text { - Habilitadores } \\
\text { da tecnologia de } \\
\text { informação }\end{array}$ & $\begin{array}{l}\text { - Aplicando as } \\
\text { técnicas de } \\
\text { implementação } \\
\text { - Avaliando as } \\
\text { condições de } \\
\text { implementação }\end{array}$ & $\begin{array}{l}\text { - A dimensão } \\
\text { dos recursos } \\
\text { humanos }\end{array}$ \\
\hline
\end{tabular}

Fonte: Extraído de Teng et al. (2006)

\section{Exemplos de aplicação e resultados obtidos}

Nesta seção, são mostrados de forma comparativa na tabela 1 a seguir os resultados da implementação de inovação - estudos de caso - em quatro organizações, citando brevemente as motivações para a adoção da inovação e seus impactos em cada organização, sendo dois os casos de sucesso e dois os de fracasso. 
Tabela 1: Comparação dos resultados das implementações da inovação de processos em algumas organizações

\begin{tabular}{|c|c|c|}
\hline $\begin{array}{c}\text { Organização e } \\
\text { Estudo de Caso }\end{array}$ & $\begin{array}{c}\text { Motivações para a } \\
\text { adoção da inovação }\end{array}$ & $\begin{array}{c}\text { Impactos da inovação } \\
\text { na organização }\end{array}$ \\
\hline $\begin{array}{l}\text { Estudo de caso de uma aplicação } \\
\text { de inovação de processos em um } \\
\text { fornecedor de porte PME - } \\
\text { pequenas e médias empresas } \\
\text { (Riley e Brown, 2001) }\end{array}$ & $\begin{array}{l}\text { - Redesenhar os processos de } \\
\text { negócio para reduzir custos; } \\
\text { - Apresentar e otimizar os } \\
\text { mecanismos de informação } \\
\text { existentes para aumentar a } \\
\text { qualidade e reduzir os riscos; e } \\
\text { - Melhorar a satisfação do cliente e } \\
\text { do empregado. }\end{array}$ & $\begin{array}{l}\text { Sucesso: } \\
\text { - Mudança cultural alinhada aos } \\
\text { objetivos da inovação; e } \\
\text { - Mudança efetiva dos processos } \\
\text { pela implementação de um sistema } \\
\text { integrado de TI. }\end{array}$ \\
\hline $\begin{array}{l}\text { Um estudo de caso de uma } \\
\text { implementação de inovação de } \\
\text { processos que desafia o paradigma } \\
\text { dos processos empresariais em uma } \\
\text { companhia do setor de eletrônica } \\
\text { (Silvestro e Westley, 2002) }\end{array}$ & $\begin{array}{l}\text { - Desenvolver as estratégias de } \\
\text { mercado (marketing); } \\
\text { - Adquirir e manter clientes } \\
\text { (vendas); } \\
\text { - Prover confiabilidade e } \\
\text { integridade } \\
\text { (operações/manutenção); } \\
\text { - Entregar produtos e serviços } \\
\text { (operações); e } \\
\text { - Calcular e coletar receitas } \\
\text { (contabilidade). }\end{array}$ & $\begin{array}{l}\text { Sucesso: } \\
\text { - Exclusão dos cargos funcionais } \\
\text { de diretores; } \\
\text { - Percepção positiva da nova } \\
\text { estrutura da companhia pela } \\
\text { gerência; } \\
\text { - Comprometimento dos } \\
\text { funcionários com as mudanças } \\
\text { implementadas pela inovação; e } \\
\text { - Organização mais orientada a } \\
\text { negócios. }\end{array}$ \\
\hline $\begin{array}{l}\text { Conflito em inovação de processos } \\
\text { em uma companhia do setor de } \\
\text { indústria de tecnologia } \\
\text { (Doswell e Sockalingam, 1999) }\end{array}$ & $\begin{array}{l}\text { - Redesenhar todos os processos de } \\
\text { negócio da organização para criar } \\
\text { uma organização mais participativa } \\
\text { e menos desigual; e } \\
\text { - Melhorar a performance } \\
\text { operacional. }\end{array}$ & $\begin{array}{l}\text { Fracasso: } \\
\text { - Falta de definição das metas de } \\
\text { custos e dos prazos-limite de } \\
\text { entrega de produtos; } \\
\text { - Manutenção da cultura } \\
\text { organizacional arcaica e da } \\
\text { estrutura funcional burocrática; e } \\
\text { - Muita resistência das pessoas à } \\
\text { implementação da inovação de } \\
\text { processos. }\end{array}$ \\
\hline $\begin{array}{l}\text { Um caso de estudo de fracasso da } \\
\text { inovação de processos no setor de } \\
\text { Telecomunicações } \\
\text { (Sarker e Lee, 1999) }\end{array}$ & $\begin{array}{l}\text { - Reestruturar os processos de } \\
\text { trabalho para melhorar a } \\
\text { performance organizacional em } \\
\text { termos de velocidade de } \\
\text { atendimento e qualidade de } \\
\text { serviços. }\end{array}$ & $\begin{array}{l}\text { Fracasso: } \\
\text { - Falta de alinhamento da inovação } \\
\text { de processos à estratégia } \\
\text { organizacional. }\end{array}$ \\
\hline
\end{tabular}

\section{Conclusão}

A inovação de processos é um termo bastante amplo e tem sido objeto de estudo de diversos autores. Neste artigo, o objetivo foi discorrer sobre os conceitos desta técnica e sintetizar as principais motivações (voltadas para a satisfação do cliente e melhoria do desempenho operacional através de preços/processos/produtos), os principais facilitadores da estrutura organizacional, da tecnologia da informação ou de recursos humanos, bem como apresentar a importância de utilizar um método e um gerenciamento coordenado da mudança provocada, como forma de melhorar o desempenho da organização e promover a criação e a manutenção de uma vantagem competitiva. Foram abordados também os resultados observados por alguns autores nas organizações que implementaram a inovação.

Mediante isto, cabe observar que o sucesso da implementação da inovação de processos está intimamente ligado à estratégia empresarial e às diretrizes estabelecidas pela organização. 
Nesta reestruturação radical das atividades do negócio, é necessário que todos estejam comprometidos com as atividades, com o desempenho e com o resultado esperado na implementação desta técnica, para atingir os objetivos almejados pela organização.

\begin{abstract}
The industrial environment of intense global competition and constant and complex changes challenges the organizations, as they need now more velocity, quality, low cost, flexibility and customer satisfaction. Their success lays upon its capacity of adequately answering to those exigencies, by continuous innovations in its products, processes and services. This work analyses the premises, the motivations and the impacts caused by Processes Innovation in organizations where this strategy is used as a way of obtaining a competitive differential in the market. To reach the intended targets, this work presents a bibliographical review of the subject and the compared results of some cases.
\end{abstract}

Key-words: organizational changes; processes innovation; organizations.

\title{
Referências
}

BERGAMASCHI, S. Um estudo sobre projetos de implementação de sistemas para gestão empresarial. São Paulo: FEA / USP, 1999.

CHIAVENATO, I. Os novos paradigmas: como as mudanças estão mexendo com as empresas. São Paulo: Atlas, 1998.

COVEY, S. Estatal é o pior negócio do mundo. Revista Exame, São Paulo, ed.609, 08/05/1996, p.33.

DAFT, R. L. Organizações: teoria e projetos. São Paulo: Pioneira Thompson Learning, 2002.

DAVENPORT, T. H. Reengenharia de processos. Rio de Janeiro: Campus, 1994.

DOSWELL, A. \& SOCKALINGAM, S. Conflict in BPR. Knowledge and Process Management. Glasgow, UK, Vol. 6, n.3, p.146-153, 1999.

HAMMER, M. Além da reengenharia. Rio de Janeiro: Campus, 1997.

HAMMER, M. \& CHAMPY, J. Reengineering the corporation. New York: HaperBusiness, 1994.

LEWIN, K. Frontiers in group dynamics: concepts, method, and reality in social science. Human relations. Vol. 1, p.541, 1947.

cross ${ }^{\text {ref }}$

MANGANELLI, R. L. Reengenharia: um guia passo a passo para a transformação da sua empresa. Rio de Janeiro: Campus, 1995.

MORRIS, D. \& BRANDON, J. Reengenharia: reestruturando sua empresa. São Paulo: Makron Books, 1994.

NADLER, D.A. \& TUSHMAN, M.L. A organização do futuro: as lições mais importantes do século XX e os próximos desafios que levarão ao novo desenho da empresa. HSM Management, n. 18, 2000.

PEREIRA, M. J. L. B. Na cova dos leões: o consultor como facilitador do processo decisório empresarial. São Paulo: Makron Books, 1999.

POIRER, C. C. Otimizando sua rede de negócios: como desenvolver uma poderosa rede de fornecedores, 
fabricantes, distribuidores e varejistas. São Paulo: Futura, 1997.

REIJERS, H. A. \& MANSAR, L. S. Best practices in business process redesign: an overview and qualitative evaluation of successful redesign heuristics. The International Journal of Management Science. London, Vol. 33, n.7, p.283-306, 2005.

RILEY, M. J. \& BROWN, D. C. Case Study of the Application of BPR in an SME Contractor. Knowledge and Process Management. Southampton, Vol. 8, n.1, p.17-28, 2001.

SARKER, S. \& LEE, A. S. IT-enabled organizational transformation: a case study of BPR failure at TELECO. Strategic Information System. Richmond, USA, Vol. 8, p.83-103, 1999.

SENGE, P.M. A dança das mudanças: os desafios de manter o crescimento e o sucesso em organizações que aprendem. Rio de Janeiro: Campus, 2000.

SILVESTRO, R. \& WESTLEY, C. Challenging the paradigm of the process enterprise: a case study analysis of BPR implementation. The International Journal of Management Science. West Midlands, UK, Vol. 30, n.15,p.215$225,2000$.

TENG, J. T. C.; GROVER, V. \& FIEDLER, K. D. Developing Strategic Perspectives on Business Process Reengineering: From Process Reconfiguration to Organizational Change. University of South California. St. Louis, EUA, Vol. 24, n.3, p.271-296, 1996.

\section{Dados dos autores:}

Débora Pereira de Macedo Soares

COPPE/UFRJ - PEP - Programa de Engenharia de Produção

R. Senador Pompeu, 119, sl. 1003 - Centro - Rio de Janeiro - RJ - Brasil - CEP: 20.221-291

Telefones: (21) 2121-8536 / (21) 9271-6513

e-mail: dmacedo@pep.ufrj.br

Rogério de Aragão Bastos do Valle

COPPE/UFRJ - PEP - Programa de Engenharia de Produção

CT - COPPE, Bl. I, sl. 236 - Ilha do Fundão - Rio de Janeiro - RJ - Brasil - CEP: 21.941-972

Telefones: (21) 2562-7848

e-mail:valle@pep.ufrj.br

Roquemar de Lima Baldam

COPPE/UFRJ - PEP - Programa de Engenharia de Produção

CT - COPPE, Bl. I, sl. 236 - Ilha do Fundão - Rio de Janeiro - RJ - Brasil - CEP: 21.941-972

Telefones: (21) 2562-7848

e-mail: roquemar@pep.ufrj.br 
Telma Dias Ragonezi

ADM Brasil Consulting

Diretoria Executiva

Consultora

Av. Afonso Pena, 3355, sl. 705 - Serra - Belo Horizonte/MG - CEP: 30.110-008

Telefones: (31) 3225-6685 / (31) 9278-0804

e-mail: telmaragonezi@admbrasilconsulting.com.br

Recebido para publicação em: 25/10/2006

Aceito para publicação em: 02/12/2006 